\title{
Multivariable Christoffel-Darboux Kernels and Characteristic Polynomials of Random Hermitian Matrices ${ }^{\star}$
}

\author{
Hjalmar ROSENGREN
}

Department of Mathematical Sciences, Chalmers University of Technology

and Göteborg University, SE-412 96 Göteborg, Sweden

E-mail: hjalmar@math.chalmers.se

URL: http://www.math. chalmers.se/ ${ }^{\sim h j a l m a r / ~}$

Received October 11, 2006; Published online December 04, 2006

Original article is available at http://www.emis.de/journals/SIGMA/2006/Paper085/

\begin{abstract}
We study multivariable Christoffel-Darboux kernels, which may be viewed as reproducing kernels for antisymmetric orthogonal polynomials, and also as correlation functions for products of characteristic polynomials of random Hermitian matrices. Using their interpretation as reproducing kernels, we obtain simple proofs of Pfaffian and determinant formulas, as well as Schur polynomial expansions, for such kernels. In subsequent work, these results are applied in combinatorics (enumeration of marked shifted tableaux) and number theory (representation of integers as sums of squares).

Key words: Christoffel-Darboux kernel; multivariable orthogonal polynomial; Pfaffian; determinant; correlation function; random Hermitian matrix; orthogonal polynomial ensemble; Sundquist's identities
\end{abstract}

2000 Mathematics Subject Classification: 15A15; 15A52; 42C05

Dedicated to the memory of Vadim Kuznetsov

\section{Introduction}

The Christoffel-Darboux kernel plays an important role in the theory of one-variable orthogonal polynomials. In the present work, we study a multivariable extension, which can be viewed as a reproducing kernel for anti-symmetric polynomials. As is explained below, our original motivation came from a very special case, having applications in number theory (sums of squares) and combinatorics (tableaux enumeration). More generally, this kind of kernels occur in random matrix theory as correlation functions for products of characteristic polynomials of random Hermitian matrices. The purpose of the present note is to highlight a number of useful identities for such kernels. Although, as we will make clear, the main results can be found in the literature, they are scattered in work belonging to different disciplines, so it seems useful to collect them in one place. Moreover, our proofs, with the interpretation as reproducing kernels, are new and conceptually very simple.

We first recall something of the one-variable theory. Let

$$
f \mapsto \int f(x) d \mu(x)
$$

${ }^{\star}$ This paper is a contribution to the Vadim Kuznetsov Memorial Issue "Integrable Systems and Related Topics". The full collection is available at http://www.emis.de/journals/SIGMA/kuznetsov.html 
be a linear functional defined on polynomials of one variable. We denote by $V_{n}$ the space of polynomials of degree at most $n-1$. Assuming that the pairing

$$
\langle f, g\rangle=\int f(x) g(x) d \mu(x)
$$

is non-degenerate on each $V_{n}$ (for instance, if it is positive definite), there exists a corresponding system $\left(p_{k}(x)\right)_{k=0}^{\infty}$ of monic orthogonal polynomials. We may then introduce the ChristoffelDarboux kernel

$$
K(x, y)=\sum_{k=0}^{n-1} \frac{p_{k}(x) p_{k}(y)}{\left\langle p_{k}, p_{k}\right\rangle}
$$

which is the reproducing kernel of $V_{n}$, that is, the unique function such that $(y \mapsto K(x, y)) \in V_{n}$ and

$$
f(x)=\int f(y) K(x, y) d \mu(y), \quad f \in V_{n} .
$$

The Christoffel-Darboux formula states that

$$
K(x, y)=\frac{1}{\left\langle p_{n-1}, p_{n-1}\right\rangle} \frac{p_{n}(x) p_{n-1}(y)-p_{n-1}(x) p_{n}(y)}{x-y} .
$$

More generally, let $V_{n}^{m}, 0 \leq m \leq n$, denote the $m$ th exterior power of $V_{n}$. It will be identified with the space of antisymmetric polynomials $f(x)=f\left(x_{1}, \ldots, x_{m}\right)$ that are of degree at most $n-1$ in each $x_{i}$. Writing

$$
\int f(x) d \mu_{m}(x)=\frac{1}{m !} \int f\left(x_{1}, \ldots, x_{m}\right) d \mu\left(x_{1}\right) \cdots d \mu\left(x_{m}\right),
$$

we equip $V_{n}^{m}$ with the pairing

$$
\langle f, g\rangle_{V_{n}^{m}}=\int f(x) g(x) d \mu_{m}(x) .
$$

Equivalently, in terms of the spanning vectors $\operatorname{det}_{1 \leq i, j \leq m}\left(f_{j}\left(x_{i}\right)\right), f_{j} \in V_{n}$,

$$
\left\langle\operatorname{det}_{1 \leq i, j \leq m}\left(f_{j}\left(x_{i}\right)\right), \operatorname{det}_{1 \leq i, j \leq m}\left(g_{j}\left(x_{i}\right)\right)\right\rangle_{V_{n}^{m}}=\operatorname{det}_{1 \leq i, j \leq m}\left(\left\langle f_{i}, g_{j}\right\rangle_{V_{n}}\right) .
$$

Every element of $V_{n}^{m}$ is divisible by the polynomial

$$
\Delta(x)=\prod_{1 \leq i<j \leq m}\left(x_{j}-x_{i}\right)
$$

The map $f \mapsto f / \Delta$ is an isometry from $V_{n}^{m}$ to the space $W_{n}^{m}$, consisting of symmetric polynomials in $x_{1}, \ldots, x_{m}$ that are of degree at most $n-m$ in each $x_{i}$, equipped with the pairing

$$
\langle f, g\rangle_{W_{n}^{m}}=\frac{1}{m !} \int f(x) g(x) \Delta(x)^{2} d \mu\left(x_{1}\right) \cdots d \mu\left(x_{m}\right) .
$$

We remark that, normalizing

$$
\Delta(x)^{2} d \mu\left(x_{1}\right) \cdots d \mu\left(x_{m}\right)
$$


to a probability distribution, it defines an orthogonal polynomial ensemble (the term is sometimes used also for the more general weights $\left.|\Delta(x)|^{\beta}\right)$. Such ensembles are important in a variety of contexts, including the theory of random Hermitian matrices [12].

An orthogonal basis of $V_{n}^{m}$ is given by $\left(p_{S}(x)\right)_{S \subseteq[n],|S|=m}$, where

$$
p_{S}(x)=\operatorname{det}_{1 \leq i \leq m, j \in S}\left(p_{j-1}\left(x_{i}\right)\right) .
$$

Here and throughout, we write $[n]=\{1, \ldots, n\}$, and we assume that the columns are ordered in the natural way. Indeed, it follows from (1) that

$$
\left\langle p_{S}, p_{T}\right\rangle=\delta_{S T} \prod_{i \in S}\left\langle p_{i-1}, p_{i-1}\right\rangle .
$$

We denote by $\Delta(x) \Delta(y) K_{m}(x, y)$ the reproducing kernel of $V_{n}^{m}$, that is, the unique element of $V_{n}^{m} \otimes V_{n}^{m}$ such that

$$
f(x)=\int f(y) \Delta(x) \Delta(y) K_{m}(x, y) d \mu_{m}(y), \quad f \in V_{n}^{m} .
$$

Equivalently, $K_{m}(x, y)$ is the reproducing kernel of $W_{n}^{m}$.

It is easy to see that

$$
K_{m}(x, y)=\frac{1}{\Delta(x) \Delta(y)} \sum_{S \subseteq[n],|S|=m} \frac{p_{S}(x) p_{S}(y)}{\left\langle p_{S}, p_{S}\right\rangle}=\frac{\operatorname{det}_{1 \leq i, j \leq m}\left(K\left(x_{i}, y_{j}\right)\right)}{\Delta(x) \Delta(y)} .
$$

Indeed, it follows from (1) and (3) that both sides satisfy (4). The equality of the two expressions can also be derived using the Cauchy-Binet formula.

The following elegant integral formula was recently obtained by Strahov and Fyodorov [29]. In Section 2 we will give a simple proof, using the interpretation of the left-hand side as a reproducing kernel.

Proposition 1 (Strahov and Fyodorov). One has

$$
K_{m}(x, y)=\frac{1}{\prod_{i=1}^{n}\left\langle p_{i-1}, p_{i-1}\right\rangle} \int \prod_{\substack{1 \leq j \leq 2 m \\ 1 \leq k \leq n-m}}\left(z_{j}-w_{k}\right) \Delta(w)^{2} d \mu_{n-m}(w),
$$

where

$$
\left(z_{1}, \ldots, z_{2 m}\right)=\left(x_{1}, \ldots, x_{m}, y_{1}, \ldots, y_{m}\right) .
$$

As a consequence, the obvious $S_{m} \times S_{m} \times \mathbb{Z}_{2}$ symmetry of $K_{m}(x, y)$ extends to a non-trivial $S_{2 m}$ symmetry.

Corollary 1. The polynomial $K_{m}(z)=K_{m}\left(z_{1}, \ldots, z_{2 m}\right)$ is symmetric in all its variables.

The motivation for the work of Strahov and Fyodorov is an interpretation of the integral (6) as a correlation function for the product of characteristic polynomials of random Hermitian matrices. The case $x=y$ is of particular interest, since (6) may then be written as

$$
\begin{aligned}
& \Delta\left(x_{1}, \ldots, x_{m}\right)^{2} K_{m}\left(x_{1}, \ldots, x_{m}, x_{1}, \ldots, x_{m}\right) \\
& \quad=\frac{1}{(n-m) ! \prod_{i=1}^{n}\left\langle p_{i-1}, p_{i-1}\right\rangle} \int \Delta\left(x_{1}, \ldots, x_{n}\right)^{2} d \mu\left(x_{m+1}\right) \cdots d \mu\left(x_{n}\right),
\end{aligned}
$$


which exhibits $\Delta(x)^{2} K_{m}(x, x)$ as a correlation function for the measure (2). In that case, the determinant formula (5) is classical, see [5, Chapter 5]. In our opinion, our proof of Proposition 1 is more illuminating than the inductive proof usually given for the special case $x=y$. For applications of Proposition 1 and for further related results, see [1, 2, 4].

An alternative determinant formula for the integral (6) may be obtained by combining two classical results of Christoffel [9, Theorem 2.7.1] and Heine [9, Theorem 2.1.2], see also [4]. We will obtain it as a by-product of the proof of Proposition 1.

Proposition 2. In the notation above,

$$
K_{m}(z)=\frac{1}{\prod_{i=1}^{m}\left\langle p_{n-i}, p_{n-i}\right\rangle} \frac{\operatorname{det}_{1 \leq i, j \leq 2 m}\left(p_{n-m+j-1}\left(z_{i}\right)\right)}{\Delta(z)} .
$$

More generally, such a determinant formula holds for the integrals

$$
\int \prod_{\substack{1 \leq j \leq m \\ 1 \leq k \leq n}}\left(z_{j}-w_{k}\right) \Delta(w)^{2} d \mu_{n}(w),
$$

but we focus on the case when $m$ is even.

The fact that the "two-point" determinant in (5) and the "one-point" determinant in Proposition 2 agree can also be derived from the work of Lascoux [15, Propositions 8.4.1 and 8.4.3], see [15, Exercise 8.33] for the integral formula in this context. Note that the reproducing property mentioned by Lascoux, and given explicitly in [17, Proposition 3], is of a different nature from (4), pertaining to integration against one-variable polynomials.

The special case of Proposition 2 obtained by subtracting the $k$ th row from the $(m+k)$ th, for $1 \leq k \leq m$, and then letting $z_{m+k} \rightarrow z_{k}$, gives the following formula for the correlation function (8).

Corollary 2. In the notation above,

$$
K_{m}(x, x)=\frac{(-1)^{\frac{1}{2} m(m-1)}}{\prod_{i=1}^{m}\left\langle p_{n-i}, p_{n-i}\right\rangle \Delta(x)^{4}} \operatorname{det}_{1 \leq i, j \leq 2 m}\left(\left\{\begin{array}{ll}
p_{n-m+j-1}\left(x_{i}\right), & 1 \leq i \leq m, \\
p_{n-m+j-1}^{\prime}\left(x_{i}\right), & m+1 \leq i \leq 2 m
\end{array}\right) .\right.
$$

Next, we give Pfaffian formulas for $K_{m}$. As is explained below, they can be deduced from results of Ishikawa and Wakayama [8], Lascoux [16], and Okada [22]. Nevertheless, we will give an independent proof, using Corollary 1. Recall that the Pfaffian of a skew-symmetric even-dimensional matrix is given by

$$
\underset{1 \leq i, j \leq 2 m}{\operatorname{pfaff}}\left(a_{i j}\right)=\frac{1}{2^{m} m !} \sum_{\sigma \in S_{2 m}} \operatorname{sgn}(\sigma) \prod_{i=1}^{m} a_{\sigma(2 i-1), \sigma(2 i)} .
$$

Proposition 3. For any choice of square roots $\sqrt{z_{i}}$,

$$
K_{m}(z)=\frac{1}{\prod_{1 \leq i<j \leq 2 m}\left(\sqrt{z_{j}}-\sqrt{z_{i}}\right)} \operatorname{pfaff}_{1 \leq i, j \leq 2 m}\left(\left(\sqrt{z_{j}}-\sqrt{z_{i}}\right) K\left(z_{i}, z_{j}\right)\right) .
$$

Moreover, for any choice of $\zeta_{i}$ such that

$$
\begin{aligned}
\zeta_{i}+\zeta_{i}^{-1} & =z_{i}+2, \\
K_{m}(z) & =\frac{\prod_{i=1}^{2 m} \zeta_{i}^{m-1}}{\prod_{1 \leq i<j \leq 2 m}\left(\zeta_{j}-\zeta_{i}\right)} \operatorname{pfaff}_{1 \leq i, j \leq 2 m}\left(\left(\zeta_{j}-\zeta_{i}\right) K\left(z_{i}, z_{j}\right)\right) .
\end{aligned}
$$


Note that (9) implies

$$
z_{j}-z_{i}=-\frac{1}{\zeta_{i} \zeta_{j}}\left(\zeta_{j}-\zeta_{i}\right)\left(1-\zeta_{i} \zeta_{j}\right)
$$

In the special case $z=(x, x)$, choosing

$$
\begin{aligned}
& \left(\sqrt{z_{1}}, \ldots, \sqrt{z_{2 m}}\right)=\left(-\sqrt{x_{1}}, \ldots,-\sqrt{x_{m}}, \sqrt{x_{1}}, \ldots, \sqrt{x_{m}}\right), \\
& \left(\zeta_{1}, \ldots, \zeta_{2 m}\right)=\left(\xi_{1}^{-1}, \ldots, \xi_{m}^{-1}, \xi_{1}, \ldots, \xi_{m}\right)
\end{aligned}
$$

Proposition 3 reduces to special cases of the identity

$$
\operatorname{pfaff}\left(\begin{array}{cc}
A & B \\
-B & -A
\end{array}\right)=(-1)^{\frac{1}{2} m(m-1)} \operatorname{det}(B-A) .
$$

The general case seems to lie deeper.

Proposition 3 is actually equivalent to Proposition 4 below, which can be deduced from known results. Indeed, rewriting (11a) using [22, Theorem 4.2] and (11b) using the case $n=m$ of [22, Theorem 4.7], we can easily see the resulting expressions to agree. More explicitly, this identity appears in [16], with a simple proof. The Pfaffian (11c) can be treated similarly, or else shown to agree with (11b) by means of a result of Ishikawa and Wakayama [8, Theorem 5.1], see Remark 3 below.

We remark that the relevant results of [8], [16] and [22] are closely related to Sundquist's identities [30], see also [6] and [7]. Moreover, (11a) is related to the Izergin-Korepin determinant for the partition function of the six-vertex model [10], which, as well as the Pfaffians in (11), has applications to alternating sign matrices [13, 14, 23, 32].

Proposition 4. Let $a_{i}$ and $z_{i}, 1 \leq i \leq 2 m$, be free variables, and let $\zeta_{i}$ be as in (9). Moreover, let $S \subseteq[2 m]$ be an arbitrary subset of cardinality $m$. Then,

$$
\begin{aligned}
(-1)^{\frac{1}{2} m(m+1)+\sum_{s \in S} s} \prod_{i \in S, j \notin S}\left(z_{j}-z_{i}\right) \operatorname{det}_{i \in S, j \notin S}\left(\frac{a_{j}-a_{i}}{z_{j}-z_{i}}\right) \\
=\prod_{1 \leq i<j \leq 2 m}\left(\sqrt{z_{i}}+\sqrt{z_{j}}\right) \operatorname{pfaff}_{1 \leq i, j \leq 2 m}\left(\frac{a_{j}-a_{i}}{\sqrt{z_{j}}+\sqrt{z_{i}}}\right) \\
=\prod_{i=1}^{2 m} \zeta_{i}^{1-m} \prod_{1 \leq i<j \leq 2 m}\left(1-\zeta_{i} \zeta_{j}\right) \operatorname{pfaff}_{1 \leq i, j \leq 2 m}\left(\frac{a_{j}-a_{i}}{1-\zeta_{i} \zeta_{j}}\right) .
\end{aligned}
$$

Note that Proposition 3 is a special case of Proposition 4 when $a_{i}=p_{n}\left(z_{i}\right) / p_{n-1}\left(z_{i}\right)$, $\left\{x_{1}, \ldots, x_{m}\right\}=\left\{z_{i}\right\}_{i \in S}$ and $\left\{y_{1}, \ldots, y_{m}\right\}=\left\{z_{i}\right\}_{i \notin S}$. Conversely, it is not hard to deduce Proposition 4 from Proposition 3 using an interpolation argument.

Remark 1. Strahov and Fyodorov found one-point and two-point determinant formulas for more general correlation functions than those of Proposition 1. In the case of

$$
\int \prod_{\substack{1 \leq j \leq m \\ 1 \leq k \leq n}} \frac{x_{j}-z_{k}}{y_{j}-z_{k}} \Delta(z)^{2} d \mu_{n}(z)
$$

their two-point formula is of the type (11a), see [29, Proposition 4.2]. Thus, Proposition 4 implies Pfaffian formulas for this correlation function. 
In [27], we need an elementary result on the expansion of the kernel $K_{m}(x, y)$ into Schur polynomials $s_{\lambda}(x) s_{\mu}(y)$. Since we have not found a suitable reference, we include it here. The proof is given in Section 4 .

Proposition 5. One has

$$
K_{m}(x, y)=\sum_{\substack{0 \leq \lambda_{m} \leq \cdots \leq \lambda_{1} \leq n-m \\ 0 \leq \mu_{m} \leq \cdots \leq \mu_{1} \leq n-m}} \frac{(-1)^{\sum_{i=1}^{m}\left(\lambda_{i}+\mu_{i}\right)}}{\prod_{i=1}^{n}\left\langle p_{i-1}, p_{i-1}\right\rangle} \operatorname{det}_{i \in[n] \backslash S, j \in[n] \backslash T}\left(c_{i+j-2}\right) s_{\lambda}(x) s_{\mu}(y),
$$

where

$$
S=\left\{\lambda_{k}+m+1-k ; 1 \leq k \leq m\right\}, \quad T=\left\{\mu_{k}+m+1-k ; 1 \leq k \leq m\right\},
$$

and

$$
c_{k}=\int x^{k} d \mu(x)
$$

Finally, let us describe our original motivation, which comes from applications that seem completely unrelated to random matrix theory. Motivated by the theory of affine superalgebras, Kac and Wakimoto [11] conjectured certain new formulas for the number of representations of an integer as the sum of $4 m^{2}$ or $4 m(m+1)$ triangular numbers. These conjectures were first proved by Milne [19, 20, 21], and later by Zagier [31]. In [25], we re-derived and generalized the KacWakimoto identities using elliptic Pfaffian evaluations. Extension of this analysis from triangles to squares leads to formulas involving Schur Q-polynomials [18] evaluated at the point $(1, \ldots, 1)$. (More precisely, these polynomials are normally labelled by positive integer partitions. Here, we need an extension to the case when some indices are negative.) Later, we realized that the resulting sums of squares formulas are equivalent to those of Milne [21]. Seeing this is far from obvious and requires an identification of the Schur $Q$-polynomials with kernels $K_{m}(z)$, where the underlying polynomials $p_{k}(x)$ are continuous dual Hahn polynomials. The key fact for obtaining this identification is the second part of Proposition 3. We refer to [26] for applications of the results above to Schur $Q$-polynomials and marked shifted tableaux, and to [27] for the relation to sums of squares.

\section{Proof of Propositions 1 and 2}

Lemma 1. Let $\phi: V_{n}^{m} \rightarrow V_{n}^{n-m}$ be def ined by

$$
(\phi f)(x)=\int f(y) \Delta(y, x) d \mu_{m}(y)
$$

Then,

$$
\left(\phi p_{S}\right)(x)=(-1)^{\frac{1}{2} m(m+1)+\sum_{s \in S} s} \prod_{i \in S}\left\langle p_{i-1}, p_{i-1}\right\rangle \cdot p_{S^{c}}(x),
$$

where $S^{c}=\{1, \ldots, n\} \backslash S$.

A corresponding statement holds when $V_{n}$ is a general $n$-dimensional vector space and $\Delta$ an element of the one-dimensional space $\left(V_{n}^{*}\right)^{n}[3, \S 8.5]$, the map $\phi: V_{n}^{m} \rightarrow\left(V_{n}^{*}\right)^{n-m}$ often being called Hodge star or Poincaré isomorphism. For completeness, we provide a proof in the present setting. 
Proof. The Vandermonde determinant evaluation

$$
\Delta(x)=\operatorname{det}_{1 \leq i, j \leq n}\left(p_{j-1}\left(x_{i}\right)\right)
$$

gives

$$
\left(\phi p_{S}\right)(x)=\sum_{\substack{\sigma:[m] \rightarrow S \\ \tau:[n] \rightarrow[n]}} \operatorname{sgn}(\sigma) \operatorname{sgn}(\tau) \int \prod_{i=1}^{m} p_{\sigma(i)-1}\left(y_{i}\right) p_{\tau(i)-1}\left(y_{i}\right) d \mu_{m}(y) \prod_{i=m+1}^{n} p_{\tau(i)-1}\left(x_{i}\right),
$$

where the sum is over bijections. By orthogonality, we may assume

$$
(\tau(1), \ldots, \tau(n))=(\sigma(1), \ldots, \sigma(m), \rho(1), \ldots, \rho(n-m)),
$$

with $\rho$ a bijection $[n-m] \rightarrow S^{c}$. One easily checks that

$$
\operatorname{sgn}(\tau)=(-1)^{\frac{1}{2} m(m+1)+\sum_{s \in S} s} \operatorname{sgn}(\sigma) \operatorname{sgn}(\rho),
$$

which gives indeed

$$
\begin{aligned}
\left(\phi p_{S}\right)(x)= & (-1)^{\frac{1}{2} m(m+1)+\sum_{s \in S} s} \frac{1}{m !} \sum_{\sigma:[m] \rightarrow S} \prod_{i=1}^{m}\left\langle p_{\sigma(i)-1}, p_{\sigma(i)-1}\right\rangle \\
& \times \sum_{\rho:[n-m] \rightarrow S^{c}} \operatorname{sgn}(\rho) \prod_{i=1}^{n-m} p_{\rho(i)-1}\left(x_{i}\right) \\
= & (-1)^{\frac{1}{2} m(m+1)+\sum_{s \in S} s} \prod_{i \in S}\left\langle p_{i-1}, p_{i-1}\right\rangle p_{S^{c}}(x) .
\end{aligned}
$$

By iteration, it follows from Lemma 1 that

$$
\phi \circ \phi=(-1)^{m(n-m)} \prod_{i=1}^{n}\left\langle p_{i-1}, p_{i-1}\right\rangle \cdot \mathrm{id}_{V_{n}^{m}} .
$$

By means of $\Delta(w, x)=(-1)^{m(n-m)} \Delta(x, w), x \in \mathbb{R}^{m}, w \in \mathbb{R}^{n-m}$, this fact can be expressed as

$$
f(x)=\frac{1}{\prod_{i=1}^{n}\left\langle p_{i-1}, p_{i-1}\right\rangle} \int f(y) \Delta(x, w) \Delta(y, w) d \mu_{m}(y) d \mu_{n-m}(w), \quad f \in V_{n}^{m} .
$$

By the uniqueness of the reproducing kernel, it follows that

$$
\Delta(x) \Delta(y) K_{m}(x, y)=\frac{1}{\prod_{i=1}^{n}\left\langle p_{i-1}, p_{i-1}\right\rangle} \int \Delta(x, w) \Delta(y, w) d \mu_{n-m}(w)
$$

This is equivalent to Proposition 1.

Remark 2. The equation (13) can also be obtained as the special case $l=n$ of the contraction formula

$$
\Delta(x) \Delta(y) K_{m}(x, y)=\frac{(n-l) !(l-m) !}{(n-m) !} \int \Delta(x, w) \Delta(y, w) K_{l}(x, w, y, w) d \mu_{l-m}(w),
$$

$0 \leq m \leq l \leq n$. Conversely, (14) follows easily from (13). 
To prove Proposition 2, we note that

$$
\Delta(x, y, w)=\frac{\Delta(x, y) \Delta(x, w) \Delta(y, w)}{\Delta(x) \Delta(y) \Delta(w)} .
$$

Applying this to (13) gives

$$
\begin{aligned}
K_{m}(x, y) & =\frac{1}{\prod_{i=1}^{n}\left\langle p_{i-1}, p_{i-1}\right\rangle} \frac{1}{\Delta(x, y)} \int \Delta(x, y, w) \Delta(w) d \mu_{n-m}(w) \\
& =\frac{1}{\prod_{i=1}^{n}\left\langle p_{i-1}, p_{i-1}\right\rangle} \frac{(\phi \Delta)(z)}{\Delta(z)},
\end{aligned}
$$

where, as in (7), $z=(x, y)$. Next we observe that, by $(12), \Delta(w)=p_{S}(w)$, with $S=[n-m]$. Lemma 1 then gives indeed

$$
K_{m}(x, y)=\frac{1}{\prod_{i=1}^{m}\left\langle p_{n-i}, p_{n-i}\right\rangle} \frac{\operatorname{det}_{1 \leq i, j \leq 2 m}\left(p_{n-m+j-1}\left(z_{i}\right)\right)}{\Delta(z)} .
$$

\section{Proof of Proposition 3}

Our main tool is the following elementary property of Pfaffians, which we learned from an unpublished manuscript of Eric Rains [24].

Lemma 2 (Rains). For arbitrary $\left(a_{i j}\right)_{1 \leq i, j \leq 2 m}$,

$$
\operatorname{pfaff}_{1 \leq i, j \leq 2 m}\left(a_{i j}-a_{j i}\right)=\sum_{S \subseteq[2 m],|S|=m}(-1)^{\chi(S)} \operatorname{det}_{i \in S, j \notin S}\left(a_{i j}\right),
$$

where $\chi(S)$ denotes the number of even elements in $S$.

For completeness, we sketch Rains' proof.

Proof. The left-hand side is given by

$$
\frac{1}{2^{m} m !} \sum_{\sigma \in S_{2 m}} \operatorname{sgn}(\sigma) \prod_{i=1}^{m}\left(a_{\sigma(2 i-1), \sigma(2 i)}-a_{\sigma(2 i), \sigma(2 i-1)}\right)=\frac{1}{m !} \sum_{\sigma \in S_{2 m}} \operatorname{sgn}(\sigma) \prod_{i=1}^{m} a_{\sigma(2 i-1), \sigma(2 i)} .
$$

Write

$$
\prod_{i=1}^{m} a_{\sigma(2 i-1), \sigma(2 i)}=\prod_{i \in S} a_{i, \tau(i)}
$$

with $S=\{\sigma(1), \sigma(3), \ldots, \sigma(2 m-1)\}$ and $\tau$ a bijection $S \rightarrow S^{c}$. Identifying $\tau$ as an element of $S_{m}$, using the natural orderings on $S$ and $S^{c}$, it is easy to check that $\operatorname{sgn}(\sigma)=(-1)^{\chi(S)} \operatorname{sgn}(\tau)$. Since the map $\sigma \mapsto(S, \tau)$ is $m$ ! to one, we obtain indeed

$$
\sum_{S \subseteq[2 m],|S|=m}(-1)^{\chi(S)} \sum_{\tau} \operatorname{sgn}(\tau) \prod_{i \in S} a_{i, \tau(i)} .
$$

The following identity appeared as [8, Theorem A.1]. 
Corollary 3 (Ishikawa and Wakayama). One has

$$
\begin{aligned}
\operatorname{pfaff}_{1 \leq i, j \leq 2 m} & \left(\frac{z_{j}-z_{i}}{a+b\left(x_{i}+x_{j}\right)+c x_{i} x_{j}}\right) \\
= & \left(b^{2}-a c\right)^{\frac{1}{2} m(m-1)} \prod_{1 \leq i<j \leq 2 m} \frac{1}{a+b\left(x_{i}+x_{j}\right)+c x_{i} x_{j}} \\
& \times \sum_{S \subseteq[2 m],|S|=m}(-1)^{\chi(S)} \prod_{j \notin S} z_{j} \prod_{\substack{1 \leq i<j \leq 2 m \\
i, j \in S \text { or } i, j \notin S}}\left(x_{j}-x_{i}\right)\left(a+b\left(x_{i}+x_{j}\right)+c x_{i} x_{j}\right) .
\end{aligned}
$$

This follows immediately from Lemma 2, by use of the Cauchy determinant

$$
\operatorname{det}_{1 \leq i, j \leq m}\left(\frac{1}{a+b\left(x_{i}+y_{j}\right)+c x_{i} y_{j}}\right)=\left(b^{2}-a c\right)^{\frac{1}{2} m(m-1)} \frac{\prod_{1 \leq i<j \leq m}\left(x_{j}-x_{i}\right)\left(y_{j}-y_{i}\right)}{\prod_{i, j=1}^{m}\left(a+b\left(x_{i}+y_{j}\right)+c x_{i} y_{j}\right)},
$$

which is reduced to its more well-known special case $a=1, b=0, c=-1$ through the elementary identity

$$
a+b\left(x_{i}+x_{j}\right)+c x_{i} x_{j}=\frac{1}{c}\left(a c-b^{2}+\left(c x_{i}+b\right)\left(c x_{j}+b\right)\right) .
$$

The proof of Corollary 3 given in [8] is more complicated.

Remark 3. The equality of (11b) and (11c) can be deduced by applying Corollary 3 to both Pfaffians, using also (10).

We only need Corollary 3 in the case when $x_{i}=z_{i}$. Then, the Pfaffian is given by

$$
\left(b^{2}-a c\right)^{m(m-1)} \prod_{1 \leq i<j \leq 2 m} \frac{x_{j}-x_{i}}{a+b\left(x_{i}+x_{j}\right)+c x_{i} x_{j}} .
$$

Indeed, one may use (15) to reduce oneself to the case $a=1, b=0, c=-1$, which is the Pfaffian evaluation in [28, Proposition 2.3].

Corollary 4. One has

$$
\begin{gathered}
\sum_{S \subseteq[2 m],|S|=m}(-1)^{\chi(S)} \prod_{j \notin S} x_{j} \prod_{\substack{1 \leq i<j \leq 2 m \\
i, j \in S \text { or } i, j \notin S}}\left(x_{j}-x_{i}\right)\left(a+b\left(x_{i}+x_{j}\right)+c x_{i} x_{j}\right) \\
=\left(b^{2}-a c\right)^{\frac{1}{2} m(m-1)} \prod_{1 \leq i<j \leq 2 m}\left(x_{j}-x_{i}\right) .
\end{gathered}
$$

We are now ready to prove Proposition 3. By Lemma 2, we have in general

$$
\operatorname{pfaff}_{1 \leq i, j \leq 2 m}\left(\left(a_{j}-a_{i}\right) K\left(z_{i}, z_{j}\right)\right)=\sum_{S \subseteq[2 m],|S|=m}(-1)^{\chi(S)} \prod_{j \notin S} a_{j} \operatorname{det}_{i \in S, j \notin S}\left(K\left(z_{i}, z_{j}\right)\right),
$$

which, by Corollary 1, equals

$$
K_{m}(z) \sum_{S \subseteq[2 m],|S|=m}(-1)^{\chi(S)} \prod_{j \notin S} a_{j} \prod_{\substack{1 \leq i<j \leq 2 m \\ i, j \in S \text { or } i, j \notin S}}\left(z_{j}-z_{i}\right) .
$$


Consider first the case $a_{i}=\sqrt{z}_{i}$. Then, by the case $a=c=0, b=1, x_{i}=\sqrt{z_{i}}$ of Corollary 4 , the sum in (16) equals

$$
\prod_{1 \leq i<j \leq 2 m}\left(\sqrt{z_{j}}-\sqrt{z_{i}}\right)
$$

This yields the first part of Proposition 3. Similarly, letting $a_{i}=\zeta_{i}$ and using (10), we can compute the sum in (16) by the case $a=1, b=0, c=-1, x_{i}=\zeta_{i}$ of Corollary 4 as

$$
\prod_{i=1}^{2 m} \zeta_{i}^{1-m} \prod_{1 \leq i<j \leq 2 m}\left(\zeta_{j}-\zeta_{i}\right)
$$

This completes the proof of Proposition 3.

\section{Proof of Proposition 5}

When $\left(e_{k}\right)_{k=1}^{n}$ is a basis of $V_{n}$, let $\left(e_{S}\right)_{S \subseteq[n],|S|=m}$ be the corresponding basis of $V_{n}^{m}$ defined by

$$
e_{S}(x)=\operatorname{det}_{1 \leq i \leq m, j \in S}\left(e_{j}\left(x_{i}\right)\right) .
$$

We then have the following general expansion formula. If $e_{i}(x)=f_{i}(x)=p_{i-1}(x)$, this is (5).

Lemma 3. Let $\left(e_{k}\right)_{k=1}^{n}$ and $\left(f_{k}\right)_{k=1}^{n}$ be arbitrary bases of $V_{n}$. Then,

$$
\Delta(x) \Delta(y) K_{m}(x, y)=\sum_{\substack{S, T \subseteq[n] \\|S|=|T|=m}}(-1)^{\sum_{s \in S} s+\sum_{t \in T} t} \frac{\operatorname{det}_{\substack{i \in S^{c}, j \in T^{c} \\ \operatorname{det}_{1 \leq i, j \leq n}}}\left(\left\langle e_{i}, f_{j}\right\rangle\right)}{\left.\left.f_{j}\right\rangle\right)} e_{S}(x) f_{T}(y) .
$$

Proof. It is enough to show that

$$
e_{U}(x)=\int e_{U}(y) R(x, y) d \mu_{m}(y), \quad U \subseteq[n], \quad|U|=m,
$$

where $R$ denotes the right-hand side of (17). Equivalently, we need to show that

$$
\sum_{T \subseteq[n],|T|=m}(-1)^{\sum_{s \in S} s+\sum_{t \in T} t} \frac{\operatorname{det}_{i \in S^{c}, j \in T^{c}}\left(\left\langle e_{i}, f_{j}\right\rangle\right) \operatorname{det}_{i \in U, j \in T}\left(\left\langle e_{i}, f_{j}\right\rangle\right)}{\operatorname{det}_{1 \leq i, j \leq n}\left(\left\langle e_{i}, f_{j}\right\rangle\right)}=\delta_{S U} .
$$

To verify this, write $U=\left\{u_{1}<\cdots<u_{m}\right\}, S^{c}=\left\{s_{1}^{\prime}<\cdots<s_{n-m}^{\prime}\right\}$, and let $X$ be the ordered sequence $\left(u_{1}, \ldots, u_{m}, s_{1}^{\prime}, \ldots, s_{n-m}^{\prime}\right)$. Consider the determinant

$$
D=\operatorname{det}_{i \in X, 1 \leq j \leq n}\left(\left\langle e_{i}, f_{j}\right\rangle\right) \text {. }
$$

On the one hand, reordering the rows gives

$$
D=(-1)^{\frac{1}{2} m(m+1)+\sum_{s \in S} s} \delta_{S U} \operatorname{det}_{1 \leq i, j \leq n}\left(\left\langle e_{i}, f_{j}\right\rangle\right) .
$$

On the other hand, applying Laplace expansion to the first $m$ rows gives

$$
D=\sum_{T \subseteq[n],|T|=m}(-1)^{\frac{1}{2} m(m+1)+\sum_{t \in T} t} \operatorname{det}_{i \in U, j \in T}\left(\left\langle e_{i}, f_{j}\right\rangle\right) \operatorname{det}_{i \in S^{c}, j \in T^{c}}\left(\left\langle e_{i}, f_{j}\right\rangle\right) .
$$

This completes the proof. 
Consider the case of Lemma 3 when $e_{i}(x)=f_{i}(x)=x^{i-1}$. Then, $e_{S}(x)=\Delta(x) s_{\lambda}(x)$, where $S=\left\{s_{1}<\cdots<s_{m}\right\}$ and $\lambda_{i}=s_{m+1-i}-i$. Thus, noting also that

$$
\operatorname{det}_{1 \leq i, j \leq n}\left(\left\langle e_{i}, f_{j}\right\rangle\right)=\operatorname{det}_{1 \leq i, j \leq n}\left(\left\langle p_{i-1}, p_{j-1}\right\rangle\right)=\prod_{i=1}^{n}\left\langle p_{i-1}, p_{i-1}\right\rangle,
$$

we obtain Proposition 5.

\section{Acknowledgements}

I thank Alain Lascoux and Eric Rains for communicating their unpublished works [16, 24], as well as for several useful comments. The research was supported by the Swedish Science Research Council (Vetenskapsrådet).

[1] Baik J., Deift O., Strahov E., Products and ratios of characteristic polynomials of random Hermitian matrices, J. Math. Phys., 2003, V.44, 3657-3670, math-ph/0304016.

[2] Borodin A., Strahov E., Averages of characteristic polynomials in random matrix theory, Comm. Pure Appl. Math., 2006, V.59, 161-253, math-ph/0407065.

[3] Bourbaki N., Éléments de mathématique, fascicule VII, livre II, chapitre III: Algèbre multilinéaire, Paris, Hermann, 1958.

[4] Brézin E., Hikami S., Characteristic polynomials of random matrices, Comm. Math. Phys., 2000, V.214, 111-135, math-ph/9910005.

[5] Deift P., Orthogonal polynomials and random matrices: a Riemann-Hilbert approach, New York University, 1999.

[6] Ishikawa M., Okada S., Tagawa H., Zeng J., Generalizations of Cauchy's determinant and Schur's Pfaffian, Adv. Appl. Math., 2006, V.36 251-287, math.CO/0411280.

[7] Ishikawa M., Wakayama M., Minor summation formulas of Pfaffians, survey and a new identity, in Combinatorial Methods in Representation Theory, Editors K. Koike et al., Adv. Stud. Pure Math., Vol. 28, Kinokuniya, Tokyo, 2000, 133-142.

[8] Ishikawa M., Wakayama M., Applications of minor summation formula III, Plücker relations, lattice paths and Pfaffian identities, J. Combin. Theory Ser. A, 2006, V.13, 113-155, math.CO/0312358.

[9] Ismail M.E.H., Classical and quantum orthogonal polynomials in one variable, Cambridge, Cambridge University Press, 2005.

[10] Izergin A.G., Partition function of a six-vertex model in a finite volume, Soviet Phys. Dokl., 1987, V.32, 878-879.

[11] Kac V.G., Wakimoto M., Integrable highest weight modules over affine superalgebras and number theory, in Lie Theory and Geometry, Editors J.-L. Brylinski et al., Progr. Math., Vol. 123, Boston, MA, Birkhäuser, 1994, 415-456.

[12] König W., Orthogonal polynomial ensembles in probability theory, Probab. Surv., 2005, V.2, 385-447, math.PR/0403090.

[13] Kuperberg G., Another proof of the alternating-sign matrix conjecture, Internat. Math. Res. Notices, 1996, V.1996, 139-150, math.CO/9712207.

[14] Kuperberg G., Symmetry classes of alternating-sign matrices under one roof, Ann. Math., 2002, V.156, 835-866, math.CO/0008184.

[15] Lascoux A., Symmetric functions and combinatorial operators on polynomials, Providence, American Mathematical Society, 2003.

[16] Lascoux A., Pfaffians and representations of the symmetric group, math.CO/0610510.

[17] Lascoux A., He S., Généralisation de la formule de Darboux-Christoffel pour les polynômes orthogonaux, C. R. Acad. Sci. Paris Sér. I Math., 1985, V.300, 681-683.

[18] Macdonald I.G., Symmetric functions and Hall polynomials, 2nd ed., Oxford, Oxford University Press, 1995.

[19] Milne S.C., New infinite families of exact sums of squares formulas, Jacobi elliptic functions, and Ramanujan's tau function, Proc. Nat. Acad. Sci. U.S.A., 1996, V.93, 15004-15008. 
[20] Milne S.C., New infinite families of exact sums of squares formulas, Jacobi elliptic functions, and Ramanujan's tau function, in Formal Power Series and Algebraic Combinatorics, 9th Conference, Vol. 3, Universität Wien, 1997, 403-417.

[21] Milne S.C., Infinite families of exact sums of squares formulas, Jacobi elliptic functions, continued fractions, and Schur functions, Ramanujan J., 2002, V.6, 7-149, math.NT/0008068.

[22] Okada S., Applications of minor summation formulas to rectangular-shaped representations of classical groups, J. Algebra, 1998, V.205, 337-367.

[23] Okada S., Enumeration of symmetry classes of alternating sign matrices and characters of classical groups, J. Algebraic Combin., 2006, V.23, 43-69, math.CO/0408234.

[24] Rains E., Quadratic Pfaffian identities, unpublished manuscript.

[25] Rosengren H., Sums of triangular numbers from the Frobenius determinant, Adv. Math., 2007, V.208, 935961, math.NT/0504272.

[26] Rosengren H., Schur Q-polynomials, multiple hypergeometric series and enumeration of marked shifted tableaux, math.CO/0603086.

[27] Rosengren H., Sums of squares from elliptic pfaffians, math.NT/0610278.

[28] Stembridge J.R., Nonintersecting paths, Pfaffians, and plane partitions, Adv. Math., 1990, V.83, 96-131.

[29] Strahov E., Fyodorov Y.V., Universal results for correlations of characteristic polynomials: Riemann-Hilbert approach, Comm. Math. Phys., 2003, V.241, 343-382, math-ph/0210010.

[30] Sundquist T., Two variable Pfaffian identities and symmetric functions, J. Algebraic Combin., 1996, V.5, $135-148$

[31] Zagier D., A proof of the Kac-Wakimoto affine denominator formula for the strange series, Math. Res. Lett., 2000, V.7, 597-604.

[32] Zeilberger D., Proof of the refined alternating sign matrix conjecture, New York J. Math., 1996, V.2, 59-68, math.CO/9606224. 